\title{
Design of intelligent power consumption optimization and visualization management platform for large buildings based on internet of things
}

\author{
Shulan Gong1,a, Yuling Wang ${ }^{1}$, Mingyu Zhang $^{1}$ \\ ${ }^{1}$ School of Information \& Electrical Engineering, Shandong Jianzhu University
}

\begin{abstract}
The buildings provide a significant contribution to total energy consumption and $\mathrm{CO} 2$ emission. It has been estimated that the development of an intelligent power consumption monitor and control system will result in about $30 \%$ savings in energy consumption. This design innovatively integrates the advanced technologies such as the internet of things, the internet, intelligent buildings and intelligent electricity which can offer open, efficient, convenient energy consumption detection platform in demand side and visual management demonstration application platform in power enterprises side. The system was created to maximize the effective and efficient the use of energy resource. It was development around sensor networks and intelligent gateway and the monitoring center software. This will realize the highly integration and comprehensive application in energy and information to meet the needs with intelligent buildings
\end{abstract}

\section{Introduction}

Globally, buildings account for almost $40 \%$ of the total end use of energy, as well as $24 \%$ of total $\mathrm{CO} 2$ emissions.[1] Domestic energy shortage, short of conventional sustainable energy supply and extensive development of energy resource led to the rapid growth of China's energy demand. At present, the energy consumption of buildings, the industrial energy consumption and the traffic energy consumption are the three main energy consumers[2], the building energyconservation is extremely urgent. The energy consumption statistical commission of CABEE(China Association of Building Energy Efficiency) released China's energy research report in 2016. It showed that the energy consumption of civil buildings in China accounts for $20 \%-25 \%$ of the total energy consumption .In 2014 the data was $19.12 \%[3]$. In the nearly 20 million square meters of the

new building every year , only $1 \%$ can be regarded as energy-efficient buildings[4]. Building energy consumption in china will continue to rise with people's living standard improved. Building energy consumption will be more than 3 times now[5]. The building energy-conservation will be huge challenge. conservation in developed countries is that the new buildings energy-conservation will reach the targets of zero energy consumption or near zero energy consumption in Euro-American countries in 2020-2030. Current situation of building energy-conservation in china is that the proportion from the new technologies and new products to applications is low. The use of a power consumption optimization and visualization management platform is designed in substantial savings in both hardware components and software.[6]The smart grid with the comprehensive energy consumption monitoring and visual management demonstration platform in the large buildings can play an important role in energy conservation.

The main contents in the article contain (1) To design intelligent sensors based on Internet of things technology to gain information parameters of power about people position and environment parameters such as temperature, humidity, illumination and power consumption by the methods of electricity information monitoring technology and detection technology. (2) To design intelligent user control terminals, including the smart socket, intelligent switch, intelligent gateway with wireless communication which can control air conditioning, lighting and other electrical appliances.(3)To design the system architecture of intelligent electricity interactive supporting platform on large buildings by components and extensible technology and massive intelligent power information mining and large scale concurrent user access processing technology (4) To design the system of demand beside response analysis control and energy efficiency optimization to monitor and control for lighting, electrical appliances, energy management and other aspects.(5) To realize electricity visualization platform, the data of power consumption is sent to the data center for statistical purposes through the network which can provide many measuring techniques based on different needs.

\footnotetext{
* Corresponding author: gongsl2008@sdjzu.edu.cn
} 


\section{The summary of the whole structure of the system.}

Intelligent power consumption optimization system and visual management platform of large buildings is a set of monitoring and control system based on Internet of things. It is set in the monitoring center of the power industry which will manage intelligent electricity and energy monitoring information of the buildings and further change the light switch. The framework of the whole system network is a sensor network connected to Internet. The monitoring center of the building can be responsible for information monitoring and related equipment control in the building which will interact information with the monitoring center of the power industry. The whole structure of the system is shown as Fig. 1

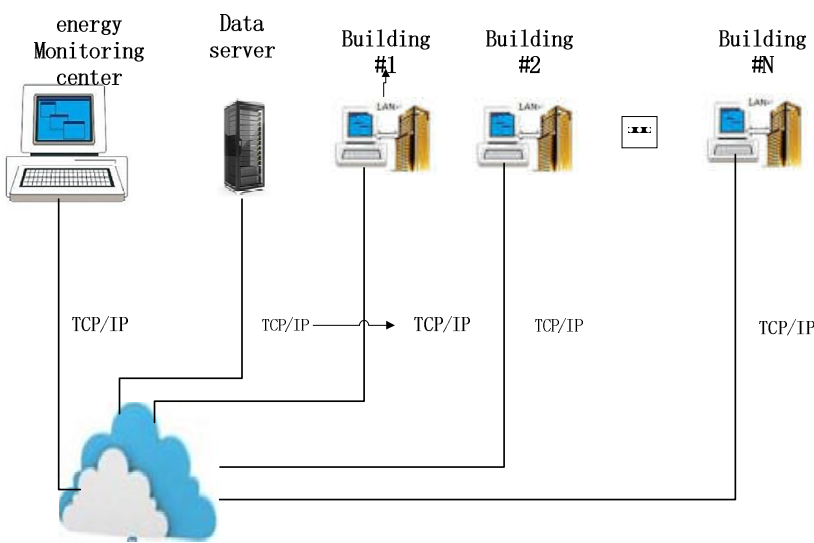

Fig.1. The overall network structure diagram of the system

The system in single building includes intelligent power consumption optimization system, visual management platform, data server, multimedia gateway in each floor, room controller, intelligent sensors, intelligent lighting system, etc. The system structure of a single large building is shown as Fig.2.

\section{The intelligent sensor}

The emerging technology of wireless sensor network(WSN) has become an increasingly feasible approach to realize control and management of building environment $[7,8,9]$.

Intelligent sensors are responsible for environmental information monitoring in intelligent power system .The single-chip MSP430F149 is used as the controller of intelligent sensors which will accomplish a remote smart home system through WIFI and Internet. Intelligen $\mathrm{t}$ sensors in this system include intelligent temperature and humidity sensors, intelligent illumination sensor, smart meter and intelligent air quality sensors. The environment variables will be converted into analogy variables through intelligent sensors, then the analogy variables will be converted into digital variables through the single-chip MSP430F149. The digital variables will be translated to the room controller through WIFI network. The room controller will exchange information with the multimedia gateway through WIFI network. The multimedia gateway has the function of inverting each other between the serial port data and the Ethernet data which will be translated to monitoring center by LAN bus. The people will observe environmental parameters and electricity consumption of each room and then realize the remote intelligent control through control command and control strategy.

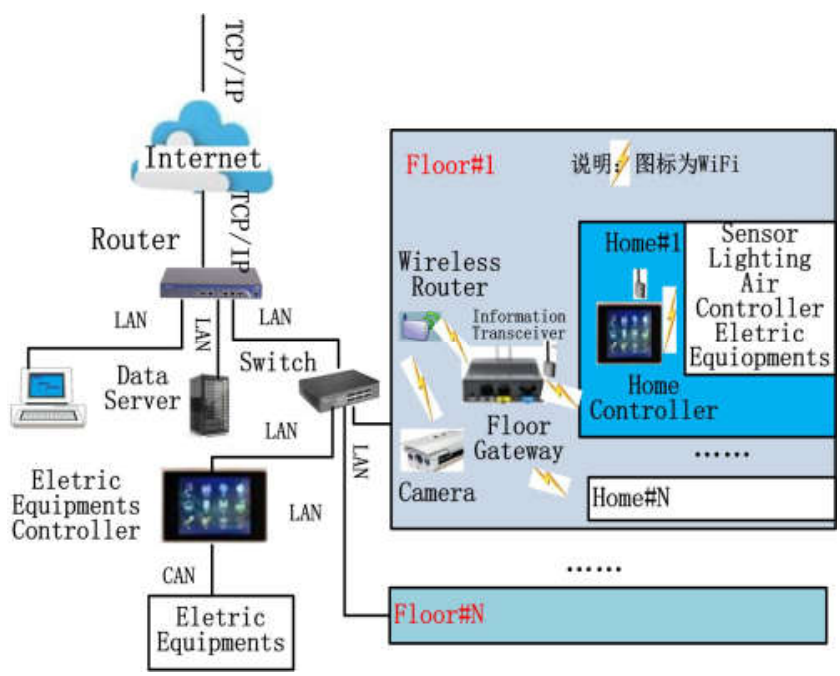

Fig.2. Tthe system structure of a single large building

\subsection{The design of intelligent temperature and humidity sensor}

The hardware part of intelligent temperature [10]and humidity sensors mainly include: $\mathrm{MCU}$ and its peripheral circuit, digital display circuit, sensor circuit, serial communication circuit, nonvolatile memory circuit, real-time clock circuit, A/D conversion circuit. MSP430F149 is used as MCU. DS18B20 is used as temperature sensor, HS1101 is used as humidity sensor.

MSP430F149 reads DS18B20 temperature value by $\mathrm{I} 2 \mathrm{C}$ bus, and then converts the value to Celsius degree which is displayed through the liquid crystal displays.In the software design of the humidity sensor module, the method scanning port is used to start the measurement at intervals of $8 \mathrm{~s}$. The measuring time length is $1 \mathrm{~s}$. The controller will count the number of pulses per unit time, determine the range of the humidity value, and display the humidity value through the LCD.

\subsection{Intelligent illumination sensor}

Intelligent illumination sensor adopts light detector with higher sensitivity and have high precision linear amplifying circuit. Its shell is designed to wallhanging Style with nice appearance. MSP430F149 is used as MCU. The master clock circuit is supplied by $8 \mathrm{MHz}$ external crystal oscillator. BH1750FVI is used as intelligent illumination sensor. 


\subsection{Smart meter}

The measurement of electric current, voltage and power in the each room can be received through the smart meter with the energy measuring chip RN8209 which will communicate with the host by SPI interface.

The hardware of the smart meter includes power module, measurement module with RN8209 chip , MCU Mega16, WIFI module ESP8226, LCD display module, external E2PROM

\subsection{The intelligent air quality sensor}

The hardware of the Intelligent air quality sensor model includes power module, measurement module with Infrared absorption sensor 6004 produced by Telaire company, MCU MSP430F149, WIFI module ESP8226, LCD display module, clock model DS1302, AD conversion module and DA conversion module. $\mathrm{CO} 2$ will be monitored by the intelligent air quality sensor in this design. Depending on the actual occupancy rate ( $\mathrm{CO} 2$ concentration), the ventilation rate is adjusted to maintain indoor air quality and the comfort of the users in order to conserve energy.

\section{The Intelligent Control of Electric Equipment}

\subsection{The intelligent switch}

The intelligent switch is used to control the lamp by manual touch or wireless control. The intelligent switch equipped with electricity metering unit is used to measure the voltage, current, power of lamp in the room. The data will be translated to room controller by WIFI. The electricity information can be viewed by user login from the remote through mobile phone. The on-off of the intelligent switch is controlled by the thyristor circuit with photo-electric coupling method. The intelligent switch circuit model mainly includes MCU module AtmelMEGA8, measurement module with RN8209, SCR control module MOC3041 and WIFI module.

The red arrow indicates the direction of power supply, the thin blue arrow indicates the flow of information, the thick blue arrow indicates the strong current of the $220 \mathrm{~V}$ in the Fig.3.

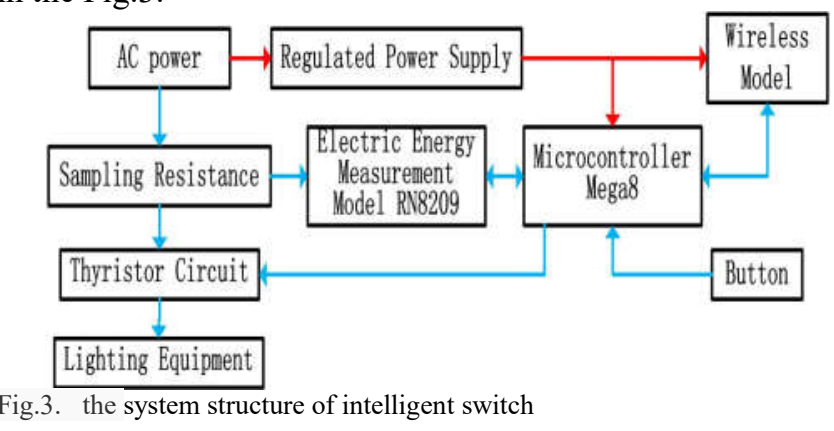

4.2 The Smart Socket
The Smart sockets are used to control household electric appliances. The user can manually control the Smart sockets through the room controller, or implement remote control it by landing room controller through the phone to control the household electric appliances on-off according to the intelligent control strategy. The Smart sockets equipped with electricity metering unit are used to measure the voltage, current, power of load in the room. Smart socket is designed in accordance with 86 boxes with the shape of the ordinary socket. The relay is used as the on-off control device of the smart socket. The triode is used as switch of the smart socket..

\section{The Room Controller}

The Room controller is designed with touch-sensitive panel and android operating system . The communication module is designed to use Socket communication technology with Java programming language. It can perform remote monitoring of the electricity consumption, power consumption and remote control of the electrical devices. The client of the room controller communicates with server by WLAN network between the sensors collecting surroundings information in the room. The data reading and assigning of the communication model is realized by JSON datainterchange format on the android platform.

The room controller can send out the communication and control instruction with simple display interface. There are command buttons on TV, lighting, air conditioner and the display interface to view temperature, humidity, light intensity and a refresh button and so on. There are two switching modes for manual mode and automatic mode. We can get the latest information about environment and the state of household appliances by the refresh button. The intelligent room controller can realize to monitor and collect the environment information in the room and then send the information to the monitoring center and the mobile phone.The room controller interface is shown as Fig.4

\section{The Smart Gateway}

The smart gateway is the core of the whole building's internal functions realizing and the hub of internal communication. Floor multimedia gateway is consisted of the wireless router and the floor gateway. The wireless router is mainly responsible for video accessing with WIFI camera and it can upload video by LAN network. The floor gateway can collect the data information from the controlled devices of each room by WIFI communication protocol and upload the data by LAN network. The information and data from LAN network is translated to the controlled devices in the room by the smart gateway and room controller. The structure of the smart gateway is showed as Fig.5. The workflow chart of the smart gateway is showed as Fig.6. 


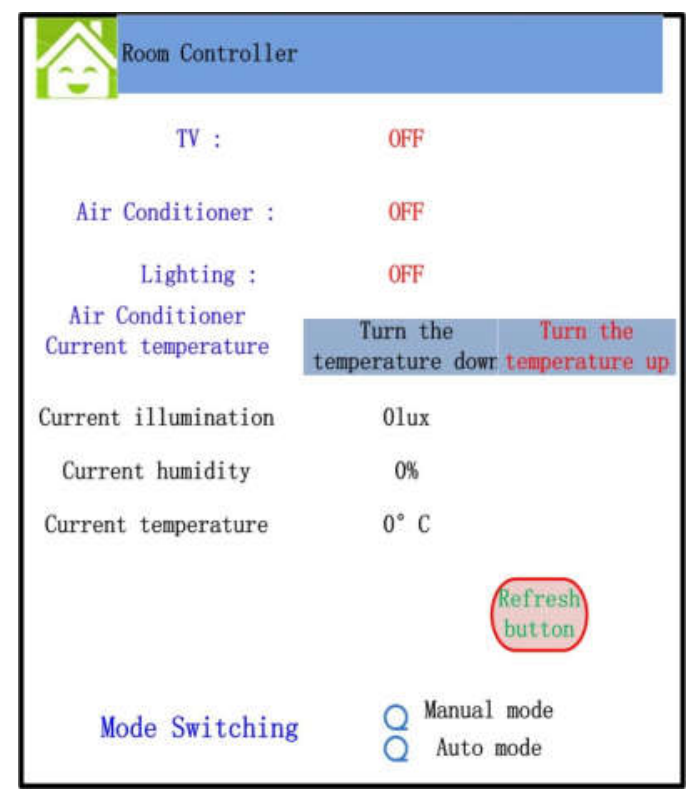

Fig.4. the room controller interface

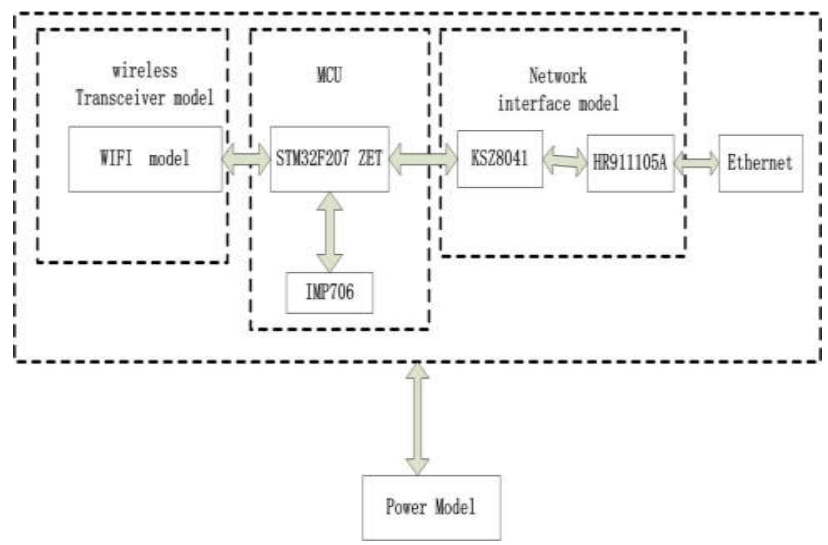

Fig.5. the structure chart of the smart gateway

\section{The Implementation of The Monitoring Center}

The monitoring center is the upper layer software of electrical power information collection and energy saving control system of the building. The monitoring center is responsible for the monitoring, controlling and data storage of the whole building. The server of the monitoring center receives and analyses the information of energy monitoring information and environment parameters from the floor gateway through TCP communication protocol. These data will be written into database at the same time. It can send control commands through the floor gateway and room controller to the controlled devices.

The main functions of the monitoring center is to implement

(1) The center monitoring software design of building energy consumption detection and control system. It includes the receiving and processing of power information, the sending of control instruction and the connection of database.
(2) Design communication protocol and algorithm to implement the network connection between the monitoring center and the smart gateway.

(3) The database design of building energy consumption detection and control system.

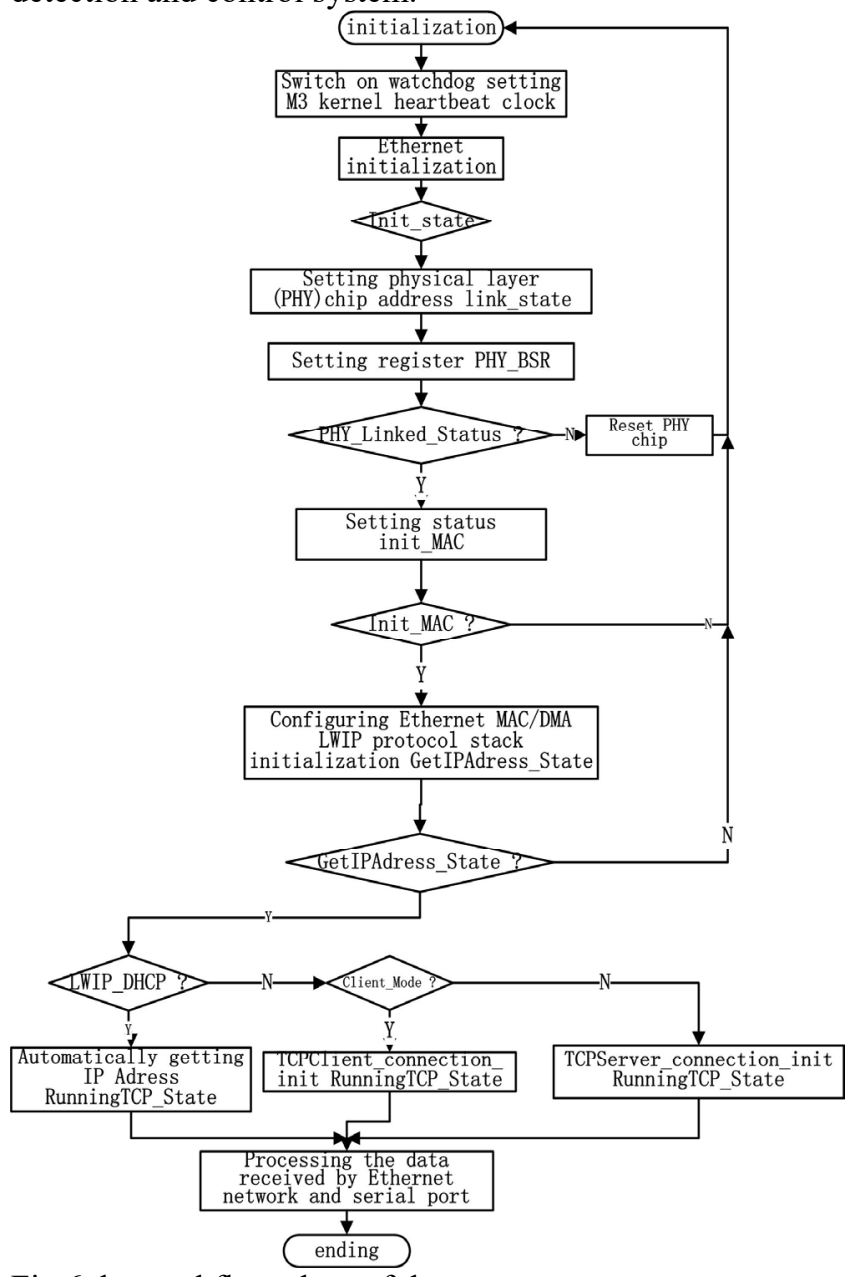

Fig.6The workflow chart of the smart gateway

According to the function of the monitoring center, the interface design of the monitoring center platform is showed as Fig.7.

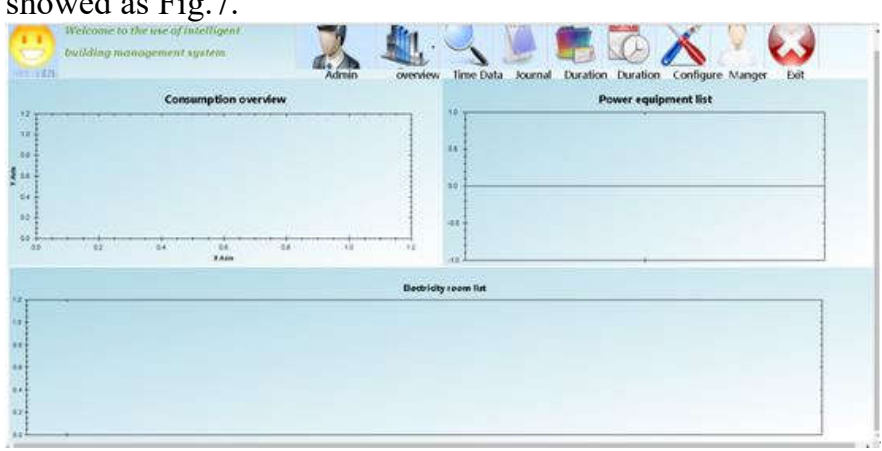

Fig. 7. the general interface of monitoring center

When the real time data button is clicked, you can look at the real energy distribution of the specific floor, it will be showed as Fig.8. When the historical time data button is clicked, you can look at the historical energy distribution of the specific floor, it will be showed as figure Fig.9. 


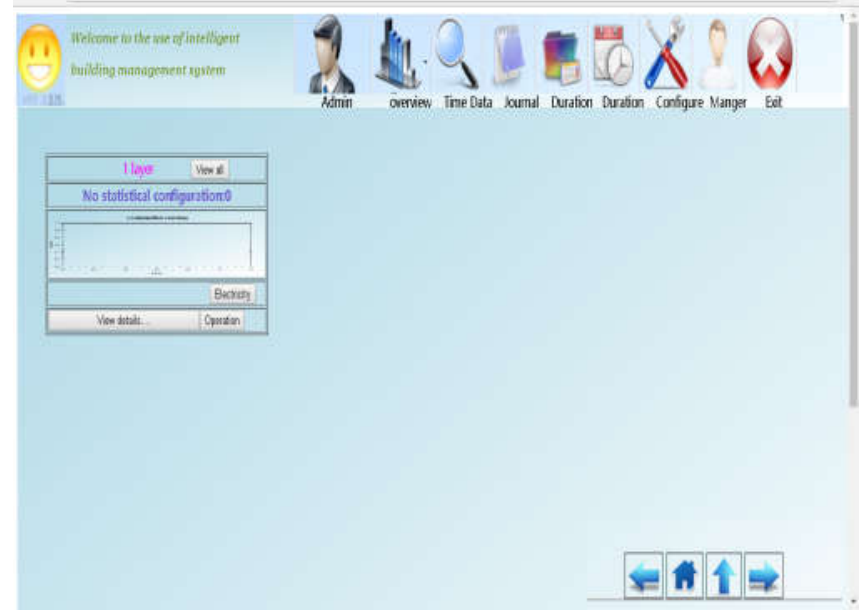

Fig. 8. the real energy distribution of the specific floor Export

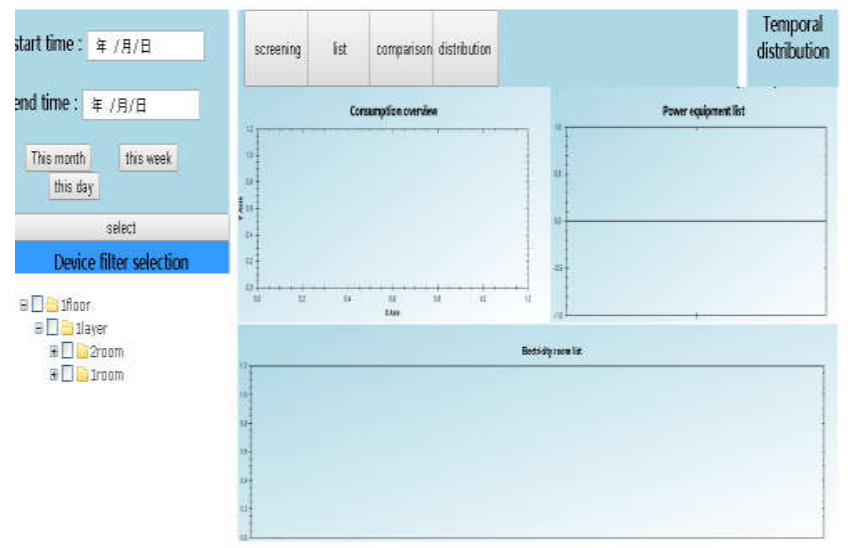

Fig.9. the historical energy distribution of the specific floor

\section{Conclusions}

The intelligent power consumption optimization and visualization management platform for large buildings based on internet of things is designed in this paper. The real-time data can be gotten by sensor network and socket communication. The parameters of air conditioner, lighting, electrical appliance equipments can be controlled by the control strategy through the remote monitoring center. The proposed system will have potential prospect in large buildings.

\section{Acknowledgment}

The work is partially supported by the key Science and Technology Planning Project of Shandong Province under Grant No.2014GGX101017 , No.2013GGX10131, No.2015GGX101024, The National Natural Science Foundation of China No.61273326. Thanks to all members of our task group. As a representative of our group, I express my sincere appreciation here to editors and specialist reviewer for your instructions and helps to the article.

\section{References}

1. Park,J.and Hong, T. Maintenance management process for reducing $\mathrm{CO} 2$ emission in shopping mall complexes .Energy and Buildings,2011,43:894-904M. Ben Rabha, M.F. Boujmil, M. Saadoun, B. Bessaïs, Eur. Phys. J. Appl. Phys. (to be published)

2. Lin Jianping . Building energy consumption is too high for comfort[N]. China Information News, 2007,11.

3. Development of China's building energy saving industry in 2016. Cnii ,2016,05.

4. Jiang Yi. Building energy consumption situation and key energy saving in China[J]. Building technology, 2007,05:26-29.

5. Guan Zhineng. Building- Maximum energy consumption black hole[N]. Economic Daily, 2007,11 .

6. Trejo-Perea,M.,et al. Local predictor for air conditioning systems using artificial neural network models for intelligent buildings. Nova Science Publishers, Hauppauge, NY, 2009a:337-351.

7. Tessa,D.,Elena,G.,James,B. Wireless sensor networks to enable the passive house-deployment experiences. European Conference on Smart Sensing Systems and Context[C], 2009:177-192.

8. Karsten,M.,Dirk,P.,Brendan,F.,etc.Towards a wireless sensor platform for energy efficient building operation. Tsinghua Science and Technology[J],2008(13):381-386.

9. Chiara ,B.,Alberto,F.,Roberto,V. An IEEE 802.15.4 wireless sensor network for energy efficient buildings. In The Internet of Things[J],2010;329338.

10. Charles,C.,Jeffrey,F.,Asad,D.,and Ruei,C.(2009). Temperature control framework using wireless sensor networks and geostatistical analysis for total spatial awareness. 10th International Symposium on Pervasive System, Algorithms, and Networks,717721. 